\title{
Transfusion-transmissible HIV infection risk in Kumba, Cameroon: A qualitative research
}

\author{
Elvis Enowbeyang Tarkang, ${ }^{1, *}$, Luchuo Engelbert Bain ${ }^{2}$ \\ ${ }^{1}$ HIV/AIDS Prevention Research Network, Cameroon (HIVPREC) PO Box 36 Kumba, Southwest region, Cameroon \\ ${ }^{2}$ Department of Military Health, Ministry of Defence, Cameroon \\ Email address: \\ ebeyang1@yahoo.com (E. E. Tarkang)
}

To cite this article:

Elvis Enowbeyang Tarkang, Luchuo Engelbert Bain. Transfusion-Transmissible HIV Infection Risk in Kumba, Cameroon: A Qualitative Research. European Journal of Preventive Medicine. Vol. 2, No. 4, 2014, pp. 38-44. doi: 10.11648/j.ejpm.20140204.11

\begin{abstract}
Transfusion-transmissible HIV remains a challenge especially where transfusion of unsafe blood is a constant practice. The study's purpose was to identify keys to unsafe blood transfusion that pose HIV transmission risk in Kumba, Cameroon. A qualitative research design was adopted, using semi-structured in-depth interviews, conducted with ten laboratory technicians working in Kumba, Cameroon, to deliberate on transfusion-transmissible HIV infection risk in their various places of work. The participants in this study perceived that there is transfusion-transmissible HIV risk in Kumba, Cameroon. The lack of safe blood means that anyone requiring blood in Kumba is at risk of transfusion-transmissible HIV infection. The establishment of blood banks in Kumba hospitals, the use of voluntary non-remunerated blood donors and the use of sensitive test kits that can detect HIV infections in the window period could improve on the quality and safety of blood donated to patients in Kumba, Cameroon.
\end{abstract}

Keywords: Blood Transfusion, HIV Transmission Risk, Voluntary Non-Remunerated Blood Donors, Blood Bank, Sensitive Test Kits

\section{Introduction}

Human Immunodeficiency Virus (HIV) and the Acquired Immune Deficiency syndrome (AIDS) remain life threatening conditions without any cure. Hence only effective preventive measures can curb this pandemic. Various strategies have been put in place to curb the global prevalence of HIV/AIDS, yet HIV/AIDS continues to spread.

More people than ever are living with HIV, largely due to greater access to treatment. At the end of 2010 an estimated 34 million people were living with HIV worldwide up $17 \%$ from 2001 . This reflects the continued large number of new HIV infections and a significant expansion of access to antiretroviral therapy, which has helped reduce AIDS related deaths, especially in more recent years [1].

Sub-Saharan Africa (SSA) remains the region most heavily affected by HIV. In 2010 , about $68 \%$ of all people living with HIV resided in SSA, a region with only $12 \%$ of the global population. SSA also accounted for $70 \%$ of new HIV infections in 2010 [1].
In Cameroon the estimated number of people living with HIV/AIDS as in 2009 was 610 000, with an adult HIV prevalence of $5.3 \%$ [2]. Here about $90 \%$ of HIV transmission occurs through heterosexual intercourse, with blood transfusion and mother-to-child transmission (MTCT) representing $10 \%$.

\subsection{Study Site}

Kumba is an urban municipal area in the Southwest region of Cameroon, with a total population of approximately 166,000 inhabitants [3]. The Republic of Cameroon is divided into 10 regions. The North West and South West regions are predominantly English speaking while the other eight regions are predominantly French speaking. The Southwest region where this study was conducted has an HIV/AIDS prevalence of 5.7\%, which is above the Cameroon national prevalence.

\subsection{Background}

Since the discovery of the first HIV case in Cameroon in 1985, there has been a steady rise in the National prevalence rate. The prevalence of HIV/AIDS in Cameroon 
rose from $0.5 \%$ in 1987 to $2 \%$ in 1992 , and $11.8 \%$ in 2002

[4]. The HIV/AIDS rate was $6.9 \%$ in 2008 [5]

The current estimated adult prevalence rate stands at $5.3 \%$ $[2,6]$, which means Cameroon is still facing a generalized epidemic of more than $3 \%$ prevalence.

Blood transfusions transmit HIV more effectively than other means, yet there has been little examination of their role in the origins and early course of AIDS in SSA. By 1955, 19 African colonies and countries reported transfusion programmes, with national rates of 718 to 1372 per 100,000 by 1964 . One million transfusions were estimated per year in SSA by 1970 and two million per year by the 1980 s, indicating that transfusions were widely used throughout SSA [7].

The special importance of blood transfusions and blood products for the transmission of HIV was recognized in both Africa and the developed world in the early years of AIDS because they transmit viral infections far more effectively than other means [8].

According to one recent study, heterosexual transmission has an efficiency of $0.01 \%$ to $0.2 \%$; unsterile injections, $0.30 \%$; and maternal-child transmission, less than $30 \%$ to $35 \%$. In contrast, blood transfusion is more than $90 \%$ efficient [9].

Despite some delays in responding to these risks, most countries now strive to protect their blood supplies [10]. But this is not always the case, especially in the developing world, where even today the blood supply is often not fully protected.

There are no blood banks in any of the health centres or hospitals in Kumba, Cameroon. Even where blood banks exist in Cameroon, the personnel are not adhering to safe practices. The situation is feared to be worse in smaller towns and villages across Cameroon, where virtually no screening or monitoring facilities are in place. The lack of awareness also means patients and their families rarely ask questions about the treatment they are receiving or the safety of blood they may need to receive.

People who need regular blood transfusion are among those most at risk of HIV transmission. However the lack of safe blood, the limited screening facilities and the widespread practice of using blood provided by drug addicts and other high risk groups means that anyone requiring blood is at risk. The growth in the number of people infected by HIV increases the danger.

Safe blood transfusion saves lives, but transfusion of unsafe blood puts lives at risk of HIV transmission. Blood which tests positive for HIV, hepatitis B, Hepatitis C or syphilis cannot be transfused and is discarded [11]

On the 10th 0f June 2011, the United Nation General Assembly, at a high level meeting on AIDS, unanimously adopted the "political Declaration on HIV/AIDS: Intensifying our efforts to eliminate HIV/AIDS", including the following new goals and targets [12]:

-Prevention.

-Universal access to treatment.

-Funding.
The declaration also includes interlinkages between HIV/AIDS and poverty, nutrition, co-infections such as tuberculosis, hepatitis and malaria, gender, maternal and child health, safe drinking water and sanitation and human rights.

However, topics such as safety of medical injections and blood products (blood transfusion) are not covered.

Cameroon is among the twenty two (22) most affected countries with HIV/AIDS in the world [12]. Of all new HIV infection, about $3.5-5 \%$ is transmitted through unsafe blood transfusions and products [13].

Safe blood saves lives but for too many patients around the world whose survival depends on blood transfusion, blood transfusion is either not available or not safe.

Every second of everyday, people around the world, of all ages and from all walks of life, need blood transfusion to survive. The reasons for transfusion vary but the demand for blood is ever-present and growing:

-Developing countries face chronic shortages of blood which particularly affect children with severe anemia due to malaria or malnutrition and women with complications of pregnancy or child birth.

-The number of accidents and injuries requiring blood transfusion is growing worldwide.

-As developing countries expand diagnostic and treatment options for cancers and blood disorders requiring transfusion, the, demand for blood is rising [11].

While the need for blood is universal, access to blood for those who need it is sadly not. The health-related Millennium Development Goals:

-To reduce child mortality.

-To improve maternal health.

-To combat HIV/AIDS infection, cannot be achieved without equitable and universal access to safe blood.

These issues are of particular concern in countries facing blood shortages like Cameroon. Globally, up to four million people have been infected with HIV by transfusion of unsafe blood [11]:

-Many countries lack policies, procedures and resources for ensuring the safety of blood, particularly in parts of Africa, including Cameroon, which are facing the HIV/AIDS pandemic.

-As some infections such as HIV cannot be detected in a person's blood during the "window period" (that is the interval between infection and detectable antibodies), laboratory testing for donated blood, no matter how sophisticated, is alone, not enough to ensure a safe blood supply. The safest blood comes from the safest blood donors.

While the researcher was in his routine work in his office a patient was referred for screening test for HIV by one clinician in the city of Kumba. The purpose of the screening was that the patient if tested negative for HIV, would donate blood to an anaemic child who had malaria. When the researcher interviewed the prospective donor, he found out that he had four wives, but the clinician who transfused the blood did not consider this fact. This finding interested the researcher and he decided on investigating 
blood transfusion procedures and HIV transmission risk in the city of Kumba, Cameroon.

\subsection{Statement of the Problem}

From the researcher's (author's) background knowledge, he was aware of the fact that information about the prevention of HIV/AIDS through safe blood transfusion is available in the city of Kumba, Cameroon. However the procedures for the selection of blood donors and the screening of transfused blood pose HIV transmission risk in the city of Kumba. The research problem was that the knowledge about the prevention of HIV/AIDS through safe blood transfusion had no impact on reducing the prevalence of HIV/AIDS in Kumba, Cameroon.

\subsection{Purpose of the Study}

The purpose of this study was to identify the keys to unsafe blood transfusion that pose HIV transmission risk in Kumba, Cameroon.

\subsection{Objectives of the Study}

The study's objective was to identify the laboratory technicians' perceptions about:

-The possibility of transmitting HIV through blood transfusion.

-The reasons for the persistent transfusion-transmitted HIV infection.

\section{Data and Methods}

The research design used for this study is a qualitative research design of phenomenological, descriptive and contextual nature.

Data collection was carried out during March 2012. Semi-structured, in-depth interviews were conducted with 10 laboratory technicians working in three different hospitals in Kumba, Cameroon.

A convenience and purposive sample was drawn from the laboratory technicians available at the time of the research, at their various places of work, where data was collected. Polit and Hungler (1997), refer to convenience sampling as the selection of the most readily available people as participants in a study [14]. In convenience sampling, participants are included in the study because they happen to be in the right place at the right time [15]. Purposive sampling involves the conscious selection by the researcher of certain participants to include in the study.

Permission to conduct the current study was obtained from the research and ethics committee of the HIV/AIDS Prevention Research Network, Cameroon (HIVPREC), a non governmental organization (NGO) for the prevention of HIV/AIDS through formalized education, working in this region of Cameroon and from the ethics committees of the establishments concerned. Participation was voluntary and informed consent was obtained from each participant to participate in the study, and that their interviews could be recorded. Human rights, anonymity (names were not used) and confidentiality were maintained through out study.

The researcher met the 10 laboratory technicians in Kumba, Cameroon and conducted a semi-structured, indepth interview with each participant about blood transfusion and HIV transmission risk in their various places of work.

Five questions were asked to the participants:

-What are the screening tests you do for blood transfusion?

-Do you believe that the blood that is transfused after screening can transmit HIV?

-How do you usually get donors for blood transfusion?

-Do you ask donor questions about their sexual behaviours before screening their blood?

-What do you think can be done to improve on the safety of blood donated to patients?

The researcher conducted the in-depth interviews and took notes, while a research assistant operated the voice-recorder, ensuring that all the discussions were captured. The data recorded during the interview were verbally transcribed and verified by comparing these statements with written notes. Coding was initially done independently by the researcher and the research assistant. The researcher then decided on the final presentation of the research results.

During data collection, the researcher probed the participants by encouraging them to explain what they perceived could be done to improve on the quality and safety of blood donated to patients, in Kumba, Cameroon. Probing is a technique used by interviewers to elicit more useful or detailed information from a participant than was volunteered in the initial reply [14]. The questions were directed at exploring their reasons, and follow-up questions were asked for clarity.

Trustworthiness refers to the ability of the researchers to convince themselves and the participants that the findings of the inquiry are trustworthy [16]. A qualitative research is trustworthy when "it accurately represents the experience of the study participants" [17]. Trustworthiness was enhanced in this study by the following:

-The researcher is an expert in the field of HIV/AIDS.

-The researcher understood the language of the participants.

-The research scrutinized the data and field notes and also reviewed literature to verify findings. The tape recorded information was also used as supporting information.

-Categorizing data, using codes and summarizing data for analysis.

\section{Research Results}

\subsection{Biographic Details}

There were six male and four female participants, and they were aged 27-55 years. All were married and having children, and were Christians. They were working in three different hospitals in Kumba, Cameroon. 


\subsection{Participant's Knowledge of the Appropriate Screening Tests for Blood Transfusion}

Safe blood transfusion saves lives but transfusion of unsafe blood puts lives at risk because HIV, hepatitis B, hepatitis $\mathrm{C}$, syphilis, malaria and other infections can be transmitted to the recipients through blood transfusion. Blood which tests positive for any of these infections cannot be transfused and is discarded [11].

All the participants in this study had knowledge of the screening tests that are to be carried out for blood transfusion, as demonstrated by this excerpt:

"The screening tests that we do for blood donation include: HIV, Hepatitis B, Hepatitis C and Syphilis. We also measure the haemoglobin level, the blood group and the rhesus, and cross-match".

\subsection{Participants' Perceptions on whether the Blood that is Transfused after Screening Can Transmit HIV}

HIV transmission through blood transfusion can be virtually eliminated through screening of all donations. In industrialized countries where all donations are screened and blood is collected from low-risk, regular donors, the residual risk of transmission come only from windowperiod infections. However in many developing countries, factors other than window period (such as poor equipment, interruption in the supply of tests, transcriptional errors, poor technicians performance) are responsible for a large proportion of unreliable results and hence the risk of transmission [18].

Screening of donated blood accounts for the majority of tests performed worldwide. As the transmission of HIV through infected blood is extremely efficient (close to $100 \%$ ), screening represents a cost-effective, preventive intervention.

All the participants in this study believed that the blood that is transfused after screening can transmit HIV.

One participant explained the situation like this:

"Yes, I believe the blood that is transfused after screening can transmit HIV because the donor can be undergoing the convectional period (window period)".

Another participant said:

"I believed the blood that is transfused after screening can transmit HIV because most of the test kits are not sensitive enough to detect HIV at its earlier stage. "There is a long sero-conversion time for HIV".

Yet another participant puts it like this:

"Yes I think it is possible that the blood that is transfused after screening can transmit HIV because of the window period that exists with this virus. An HIV positive donor may be negative during the window period (the interval between infection and detectable antibodies)".

\subsection{How Participants got Donors for Blood Transfusion}

In many resource-limited countries like Cameroon, blood transfusion services are still mainly hospital-based; blood is usually collected when required and therefore stored blood stocks are minimal or non-existent. Blood banks are non existent. Rapid screening attracts donors who wish to use the transfusion service to find out their HIV status. Such donors represent an obvious threat to blood safety as they are more likely to be at higher risk of HIV infection [18].

Too many countries including Cameroon, still reply on family replacement (a member of the patient's family donating his/her blood) or paid donors. Family replacement donors may feel under pressure to donate and may therefore hide aspects of their health and lifestyle, which could mean that their blood is more likely to contain infections. In the case of paid donors, government may think that the financial incentive will motivate more donation and boost supplies, but paid donors are often pushed by need and are therefore also more likely to avoid mentioning important details about their health status [11].

The participants in this study said they get their donors from family members of the patients or they look for donors around, as demonstrated by this excerpt:

"We ask the patient to come with his/her relatives for the screening. At times we look around for people with the same blood group as the patient".

Another participant put it like this:

"It is the responsibility of the patient's family to provide us with donors. We ask the families to provide us with a donor. Most families provide donors from the families. So more often than not, the patients'families bring us donors".

\subsection{Participants' Opinions on whether they Ask Donors Questions about their Sexual Behaviours before Screening their Blood}

In many resource-limited countries like Cameroon blood transfusion services are still mainly hospital-based, blood is usually collected when required [18]. Rapid screening attracts donors who represent an obvious threat to blood safety as they are more likely to be at higher risk of infection.

As mentioned earlier, family replacement donors may feel under pressure to donate and may therefore hide aspects of their health and lifestyle, which could mean that their blood is more likely to contain infection. Paid donors are often pushed by need and are therefore also more likely to avoid mentioning important details about their health status.

The prevalence of HIV infection among family replacement donors and paid donors is generally the same as in the general population [11].

Participants in this study said they do not ask donors about their sexual behaviours before screening their blood, as demonstrated by this excerpt:

"No, most of the situations one is faced with are desperate situations that one turns to minimize that aspect of sexual behaviours, especially with the absence of a blood bank".

Another participant put it like this:

"No, because I believe the question is traumatizing and already gives a sense of stigmatization. Also, the patient too should be able to do his/her findings". 
Another participant said:

"No! But we make them know the screening tests we have to do before donating blood, because you will know what to tell them during post-test counseling, if they are tested positive for HIV'.

\subsection{Participant $S$ ' Opinions of what Can be Done to Improve on the Quality and Safety of Blood Donated to Patients}

The response to the question "what do you think can be done to improve on the quality and safety of blood donated to patients", were categorized according to the key elements that address blood supply adequacy and transfusion safety, outlined by the WHO [19]:

-Voluntary non-remunerated blood donor.

-Blood banking.

-Highly sensitive test kits.

\subsubsection{Voluntary Non-Remunerated Blood Donors}

Voluntary blood donation programme is the foundation for safe and quality blood transfusion service as the blood collected from voluntary non-remunerated blood donors is considered to be the safest.

A voluntary non-remunerated blood donor is a person who gives blood, plasma or other blood components of his/her own free will and receives no payment for it either in the form of cash or in-kind which could be considered a substitute for money. Their donation is an altruistic gift and not a marketable commodity $[11,20]$. They are the key to ensuring that safe blood is available to every patient who needs it wherever they may be.

Evidence around the world shows that the prevalence of $\mathrm{HIV}$ infection and transfusion-transmissible infections is invariably lowest among voluntary unpaid donors who give blood for purely altruistic reasons. Voluntary donors are more likely to be honest in answering the questions about their health and lifestyles that help to screen out those at risk of carrying these infections. They are also more likely to lead low-risk lifestyles, benefiting both themselves and the patients who receive their blood $[11,20]$.

The goal of voluntary blood donation is to wipe off the scarcity of blood and ensure availability of safe and quality blood and other blood components, round the clock and throughout the year. This will lead to alleviation of human sufferings, even to the far-flung remote areas in the country.

Voluntary blood donation has the following objectives:

-Provide safe and quality blood and blood components collected from voluntary donors, round the clock, at affordable cost to the general public and free of cost to the poor.

-Ensuring safety and quality of blood.

-Motivate and maintain a permanent well-indexed record of voluntary blood donors.

-Educating the community on the beneficial aspects of blood donation and harmful effect of collecting blood from paid donors.

-Actively encourage voluntary blood donation and gradually eliminate professional blood donors.

-Promote AIDS awareness and education to the general public.

-Assists the various organizations, clubs, colleges, public and private institutions and the public to conduct voluntary blood donation drives and arrange for motivational talks to enable progressively increase in the number of voluntary non-remunerated blood donors every year [20]. Any healthy adult, both male and female, can donate blood. Men can donate safely once in every three months while women can donate every four months.

In every country, a reliable supply of safe blood from donors with different blood groups is needed throughout the year. It is therefore crucial that healthy, voluntary, unpaid blood donors make a commitment to give blood regularly.

In addition to ensuring an adequate supply of blood at all times, regular voluntary blood donors are the safest donors because they have been educated about how to stay healthy and lead lifestyles that are free from the risk of acquiring serious infections.

The participants in this study believe that the utilization of voluntary non-remunerated blood donors will improve on the safety of blood donated to patients as demonstrated by this excerpt:

"First and most importantly, blood transfusion should be discouraged. But if blood transfusion is the only option, I believe the utilization of voluntary unpaid blood donors is the best choice. But as I said, hospitals should try by all means to limit blood transfusion".

\subsubsection{Blood Banking}

Blood bank/blood centre is defined as a building or location specifically dedicated to blood collection, component production, testing, storage, and distribution. In a blood centre, the space allocation, type and number of equipment, amount of consumables, and the number and category of staff will vary with the quantum of blood collected and processed per annum. The scope of the centre will also depend on the size, local requirements and modus operandi [20].

Large hospitals with attached medical colleges or specialized medical institutions should have centres with all facilities to ensure adequate and efficient provision of blood. The small hospital-based centres can receive their blood supplies from regional or larger hospital centres in the area and may be provided only basic laboratory facilities for controlled storage of whole blood and blood components.

Basic functions of a blood transfusion centre are:

- Organizing the service.

- Recruitment of donors.

- Collection, processing, storage and distribution of blood and blood components.

- Laboratory investigations.

- Participation in clinical use of blood and blood components.

- Teaching and training. 
- $\quad$ Research and development.

Everybody involved in donor recruitment should value voluntary, unpaid donors as the source of a sustainable and safe blood supply. Regardless of the type of national blood programme, whether hospital-based or coordinated at national or regional levels, the common focus should be the recruitment and retention of voluntary unpaid donors [11, 21].

The participants in this study believe that the introduction of blood banks in the hospitals will improve on the quality of blood donated to patients in Kumba, Cameroon.

One participant explained the situation like this:

"There should be the establishment of blood banks in every hospital where transfusion is carried out, and the services should run 24 hours a day".

Another participant said:

"It is important that blood banking system should be instituted in all the sub-divisions of Cameroon; and due to the window period of HIV, blood stored for long in the bank could be rescreened for HIV'.

\subsubsection{Highly Sensitive Test Kits}

Screening of donated blood accounts for the majority of HIV tests performed worldwide. As the transmission of HIV through infected blood is extremely efficient (close to $100 \%$ ), screening represents a highly cost-effective, preventive intervention [18]

The diagnosis of HIV infection has traditionally been made by detecting antibodies against HIV, the most commonly used method being ELISA.

ELISA tests are designed for batch testing (that is, screening 96 to several hundred specimens per day), making them suitable for use in surveillance and centralized blood transfusion services. The validity of the ELISA results depends on skilled technicians who are able to prepare correctly the necessary reagents, pipepette with accuracy and operate the equipment.

The conditions required to perform ELISA cannot be met in all parts of the world and there was a need to develop HIV test for the use in laboratories with limited facilities. Advances in technology have led to the development of a wide range of simple/rapid tests, based on the following principles: agglutination tests, Immunocomb tests, Immunodot tests, using flow-through membranes and most recently, immunochromatographic membrane tests.

Most simple/rapid tests are presented in a kit form that requires no other reagent or equipment. As the procedures are very easy, there is less chance of error and they can be carried out by staffs with no formal laboratory training. The interpretation of the test results is generally straight forward and many simple/rapid tests include an internal control which validates the test result. Preliminary results can be obtained within a few minutes. Some simple/rapid tests do not require refrigeration and can be stored at temperatures between $2^{\circ} \mathrm{c}$ and $30^{\circ} \mathrm{c}$ [18].
Assays for screening of donated blood need to be highly sensitive to detect infections as early as possible (window period) and should be able to detect a broad range of HIV1/HIV2 viruses and variants.

Participants in this study believe that the introduction of very sensitive and reliable tests for HIV1/2 that can detect possible window period infection will improve the quality of blood donated to patients as demonstrated by this excerpt:

"Reagents that are very sensitive to detect HIV infections even in the window period should be provided".

Another participant put it like this:

"Quality tests that can diagnose early HIV infection should be provided".

\section{Discussion of Research Results}

The participants (laboratory technicians) in this study perceived that the blood that is transfused to patients after screening could transmit HIV.

Their perceptions were based on the following:

-There are no blood banks in any of the clinics or hospitals in Kumba, Cameroon.

-Regular voluntary, non-remunerated blood donors, who are considered to be the safest donors, are not used in Kumba. Instead family replacement donors (family members) and paid donors are used most of the time. Family replacement donors and paid donors may feel under pressure to donate and are often pushed by need, and may therefore hide aspects of their health and lifestyle; which could mean that their blood is likely to contain infection.

Evidence from around the world shows that the prevalence of HIV infection and other transfusiontransmissible infections is invariable lowest among voluntary unpaid donors who give blood for purely altruistic reasons. Voluntary donors are more likely to be honest in answering the questions about their health and lifestyle that help to screen out those at risk of carrying these infections.

-Non-availability of reagents and test kits that are very sensitive to detect HIV infections even in the window period.

Assays for screening of donated blood need to be highly sensitive to detect infections as early as possible (window period) and should be able to detect a broad range of HIV1/HIV2 viruses and variants.

\section{Conclusion}

The lack of safe blood, the limited screening facilities, and the widespread practice of using blood provided by drug addicts and other high risk groups, and the nonavailability of blood banks, means that anyone requiring blood in the city of Kumba is at high risk of transfusiontransmissible HIV infection. The growth in the number of people infected by HIV increases the dangers. 


\section{Recommendations}

The following recommendations were made from this study

-The establishment of blood banks in all the hospitals and health centres in Kumba, Cameroon.

-The use of regular voluntary non-remunerated blood donors.

-The availability of very sensitive reagents and test kits that can detect HIV infections even in the window period.

All of these strategies will assure the safety of blood donated to patients who require blood transfusion.

\section{References}

[1] UNAIDS, World AIDS Day Report: How to get to Zerofaster, smarter, better. Geneva, Switzerland, 2011.

[2] UNAIDS, Report on the global AIDS epidemic. Geneva, Switzerland, 2010.

[3] Bureau Central des Recensements et des etudes de population (BCREP), Livre "Rapport de Presentation. Cameroon, 2010.

[4] International Monetary Fund, Cameroon: poverty reduction strategy paper. IMF country Report No. 03/249. August 2003. Washington DC: IMF.

[5] Central Intelligence Agency (CIA), Cameroon, Cameroon HIV/AIDS-adult prevalence rate, 2008. From: $\mathrm{http} / / /$ indexmundi.com/cameroon/ (accessed on 23/08/2008).

[6] UNICEF, UNAIDS, and WHO, Young people and HIV/AIDS: opportunity in crisis. New York: UN, 2009. From:

http://www.unicef.org/infobycountry/Cameroon_statistics.ht ml\#76 (accessed on 15/08/2011).

[7] W. H. Schneider, and E. Drucker, Blood transfusion in the early years of AIDS in sub-Saharan Africa, Am J Public Health, vol. 96(6), pp. 984-994, 2006.

[8] J. M. Mann, Human immunodeficiency virus seroprevalence in pediatric patients of 2 to 14 years of age at Mama Yemo in hospital, Kinshasa, Zaire, Pediatrics, vol. 78,pp. 673-677, 1986.

[9] P. Piot, and M. Bartos, "The epidemiology of HIV and AIDS" in M. Essex, S. mboup, P. J. Kanki eds, AIDS in Africa, $2^{\text {nd }}$ ed: 202-204. Kluiver, New York, 2002.
[10] WHO, Blood safety and clinical technology, strategy 20002003. Geneva. WHO, 2001. (Available at: http://www.who.int/entity/injection/safety/about/strategy/en/ BCT strategy.pdf accessed March 6th 2012).

[11] WHO, IFRC, FLODS, and ISTB, World Blood Donor Day. "Celebrating your gift of blood". Making the most of world blood donor day, June 142007.

[12] L. K. Tammy, V. Lars, E. R. Zoe, and G. Gretchen, Special Report for the UN high-level meeting on AIDS, 8/10 June 2011: HIV/AIDS in the context of other global challenges. Global 2015. Berlin, Germany, 2011.

[13] AVERT, Blood safety and HIV, 2011. Available from http://www.avert.org/blood-safety-hiv.htm. (Accessed on $12 / 10 / 2011)$.

[14] D. F. Polit, and B. P. Hungler, Nursing research: principles and methods. $4^{\text {th }}$ edition. Philadelphia: Lippincott, 1997.

[15] N. Burns, and S. K. Grove, Understanding nursing research; $2^{\text {nd }}$ edition. Philadelphia: Saunders, 1999.

[16] E. Babbie, and J. Mouton, The practice of social research. Oxford: Oxford University Press, 2004.

[17] H. J. Streubert, and D. R. Carpenter, Qualitative research in nursing: advancing the humanistic imperative. $2^{\text {nd }}$ edition. Philadelphia: Lippincott, 1999.

[18] WHO, Weekly Epidemiological Record No 42. 73: 321-328, Geneva: WHO, $16^{\text {th }}$ October 1998.

[19] WHO, Blood safety key global facts and figures in 2011. Switzerland, Geneva: WHO, 2011. (Available at http://www.who.int/worldblooddonorday/media/who blood _safety_factsheet_2011.pdf. Accessed November 15, 2011).

[20] K. S. Rao, National AIDS Control Organization. Voluntary Blood Donation Programme: An operational Guideline. Ministry of Health and Family Welfare. Government of India, New Delhi, 2007.

[21] CDC, Morbidity and Mortality Weekly Report: progress towards strengthening national blood transfusion services in 14 countries, 2008-2010. MMWR No 60/46, USA, November 25, 2011. USA. 